\title{
Semantic Parsing with LFG and Conceptual Representations
}

\author{
Rodolfo Delmonte \\ Department of Linguistics and language Teaching Theory, Università di Venezia, \\ Ca' Garzoni-Moro, San Marco 3417, 30124 Venezia, Italy \\ E-mail address: delmonte@IVEUCC.BITNET
}

\begin{abstract}
A semantic parser is presented which aims at the attainment of a high linguistic cov erage and an extendibility to many languages. To this end, a frequency dictionary for Italian has been classified following LFG theoretical framework and a general decoder has been implemented to pass from the syntectic to the semantic and conceptual levels. Syntactic structural derivation is produced by complete lexical forms, lexical redundancy rules and an extended phrase structure grammar which has been implemented in Prolog using XGs Semantic and conceptual representations are produced following Jackendoff's system of conceptual representations plus a number of additions to compute time reference mainly inspired by J.Allen's system (1983a,b). We believe that a text rhetorical organization is mainly governed by linguistic principles like the alternation of FOCUS and TOPIC, the use of definiteness to qualify referring expressions, etc. We describe two algorithms to analyse the level of text: Logical Form, which provides scope assignment to quantified expressions; an algorithm for anaphora resolution which incorporates linguistic information and a number of psycholinguistic heuristics. Finally, we briefly describe how the inference engine provided by KL-One is integrated into the previous modules to produce semantic entailment and linguistic inferences.
\end{abstract}

Key Words: LFG theory, functional representation, lexical representation, Prolog parsing, logical form, conceptual representation, meaning \& inference, KL-One, anaphora resolution, time reference representation.

\section{The Lexical Level}

A set of computer programs building on a primitive description of lexical items based on categorial status, subcategorization frames, inherent features, syntactic and semantic classes produces a number of representations divided up into four levels: a syntactic level, or Level 1, a functional level Level 2; a thematic level Level 3; and a conceptual level Level 4. Each level builds on the lower one using restrictions based on the information present at that level and the class information available. For instance, syntactic classes are only available at Level 2 in order to assign grammatical functions; semantic classes are only available at Levels 3 and 4 in order to build thematic roles and conceptual representations. As to conceptual representations, they are an expansion of thematic roles, which are abstractions and reductions on the finer grained semantic structures of predicates, and can likewise be produced with additional information constituted by semantic classes.

\section{1,1 The classification}

The lexicon we have implemented is taken from the Frequency Dictionary of Italian with various additions: it contains over 7000 lemmas. There are approximately 4000 nouns, 1000 adjectives and 2000 verbs.

Rodolfo Delmonte is Associate Professor of Applied Linguistics at the University of Venice. He received his doctoral degree in 1973 in Venice: he was then granted the Australian-European Award and studied in Melbourne where he received the Ph.D. degree in 1978 from Monash University. At the beginning, he worked in quantitative linguistics mainly in literary research; he was then involved in a number of major projects transferring prosodic and phonological rules of Italian into implementations for synthetic speech. Since 1983 he has been more interested in parsing both syntactic and semantic: he is lately working on an EUREKA man-machine interface project for the automatic generation of linguistic descriptions. 
In addition, we have a list of about 500 function words(see Delmonte, 1989). The basic classification is intended to provide information to be used by more than one level of representation. Level 1 is a complete syntactic representation with a rich number of features partly used by Level 2, partly by Level 3, partly by lexical redundancy rules, and partly by Level 4 . Some of these features are associated with the predicate and some with its arguments: the former are features such as [+RAIS], [+INAC], [+PERC], [+FACT]; the latter are inherent semantic features and include the following, [+ANIM, -ANIM, +HUM, -HUM, +ABST, ABST]. Only three features can have agentive and causative meaning, [+ANIM, +HUM, -HUM], where +Anim, stands for animal kind, +Hum for human kind, and -Hum for natural powers. These three features individuate then possible causers, the first two intentional, and the latter non-intentional. Level 1 lexical classification is obtained by an interactive program written in $\mathrm{C}$ language, which presents a series of windows with multiple choice menus. The classifier - a linguist - is thus required to provide what we define as full subcategorization frames which basically expand the classical notion of syntactic subcategorization and extend it to include explicit reference to the subject argument, a distinction between argumental XP arguments and predicative XP arguments, plus a number of syntactic and semantic features which are meant to allow the translation of each level of representation. They are also used by the parser to impose restrictions on the class of semantically compatible and appropriate modifiers and adjuncts.

Level 2 is an annotation in lexical-functional grammar (LFG) terms of complete grammatical functions onto major syntactic constituents: this level is coupled with a number of lexical redundancy rules, which are expressed as rules in the parser, in order to build derived structures such as passivized and pronominalized structures, inchoativized and intransitivized structures and so on.

Level 3 is a translation into the thematic roles augmented by a system of aspectual features. Aspectual classes are accounted by five features: STATES, ACTIVITIES, ACHIEVEMENTS, ACCOMPLISHMENTS, MOMENTANEOUS. They are derived from the more primitive features: -imperative, \pm imperative, +imperative, quasi imperative and the interaction with syntactic classes. In turn, both syntactic classes and aspectual classes contribute to the labelling of arguments in terms of Theta-roles as appearing in Level 3 representations. In terms of thematic relations, the subject of activity verbs cannot be a Theme and can be either an Agent or an Experiencer. In the class of activity predicates, achievements can be distinguished from accomplishment in that the latter class requires an agentive subject whereas the former does not. It is part of the nature of accomplishments that they involve a result or an end, and these can be expressed by the direct object or by another argument strictly subcategorized by the verb. Thus, an activity verb like "run" becomes an accomplishment if there is a specific distance to overcome or a specific goal to attain. This implies that verb aspectual classes are closely connected with the semantics of the arguments of a predicate. As to syntactic classes, PSYCHic verbs receive a labelling of their arguments in terms of Theta-roles that assigns THEME to the Subject argument and EXPERIENCER to the Object one. Level 4 is a classification in conceptual representation derived strictly from Level 3 plus information on semantic categories. Level 1 lexical classification is listed in the following Table which shows the basic items included in our system of representation:

\section{1. verb:}

1.1. verbal class: 1.transitive, 2.inaccusative, 3.inergative, 4.reflexive, 5.copulative, 6.inherent reflexive, 7.impersonal, 8. psych1, 9. psych2;

1.2. syntactic-aspectual features: 1.raising, 2.perception, 3. \pm imperative, 4.imperative, 5.non-imperative, 6.quasi-imperative; 
1.3. interpretive-semantic classes: 0. extensional, 1.subjective/intensional, 2.hyperintensional, 3.quantity/modality, 4.factive, 5.emotional/affective, 6.properties, 7.mental activities, 8.inchoatives, 9.measure;

1.4. arguments of the predicate: 1.4.1 syntactic classes: NP, AP, PP, VP', S', PPpred, NPpred, APpred, AdverbSN; 1.4.2 preceding particles: all prepositions

[1.A 2.DA 3.DI 4.IN 5.SU 6.CON 7.PER 8.TRA 9.CONTRO a.VERSO b.DA_ PARTE_(DI) c.DAVANTI_A d.INTORNO_A e.(MATTER) IN, DI, f.(LOCAT) IN,DI,A]

1.5 semantic features of predicate-argument structures: 1.5.1. inherent features of obligatory arguments (NPs, APs, PPs) of the predicate: $\{$ [+ANIM], [-ANIM], [+HUM], [-HUM], [+ABST], [-ABST], [LEXICALLY SPECIFIED], [NONE], [-NUM], [ALL], [ PLUR]\} (either one or another in a set); 1.5.2. $\mathrm{VP}^{\prime}=$ subject control $\{\mathrm{SUBJ}, \mathrm{OBJ}, \mathrm{OBJ} 2\} ; 1.5 .2 .1$. syntactic feature [+ restruct]; 1.5.3. S' = subject control $\{\mathrm{SUBJ} / \mathrm{X}, \mathrm{SUBJ}, \mathrm{X}\} ;$ 1.5.3.1 propositional $\{$ excl, quest, prop $\} ; 1$ 1.5.4. XPpred $=$ subject control by a lexical redundancy rule associated with each predicative argument

\section{2. adjectives:}

2.1. typology: 1.predicative, 2.attributive, 3.predicative/attributive, 4."tough" predicates, 5.transitive, 6.factive;

2.2. syntactico-semantic properties: 1.imperative, 2.non-imperative, 3.quasi-imperative, 4.inaccusative;

2.3 semantic properties: 0.state, 1.measure, 2.origine, 3.quantity/modality, 4.time, 5.evaluating, 6.colour, 7.intensional, 8.hyperintensional;

2.4. arguments of the predicate: as for verbs

\section{3. nouns:}

3.1. nominal classes: 0.pure nominal, 1.transitive, 2.inaccusative, 3.inergative, 4.reflexive, 5.copulative, 6.ambiguous(active/passive), 7.impersonal, 8. psych, 9.passive

3.2. typology: 0.proper nouns(only +human), 1.geographical, 2.common

3.2.1.locative, 2.2.quantity/modality, 2.3.temporal, 2.4.legal institution, 2.5.profession, 2.6.general,

2.7.species, 2.8.relationals, 2.9.events, 2.0.subjective;

3.3. inherent features, as for arguments of the predicate; 3.4. arguments of the predicate as for verbs

We report below tables and excerpts from Delmonte, 1989

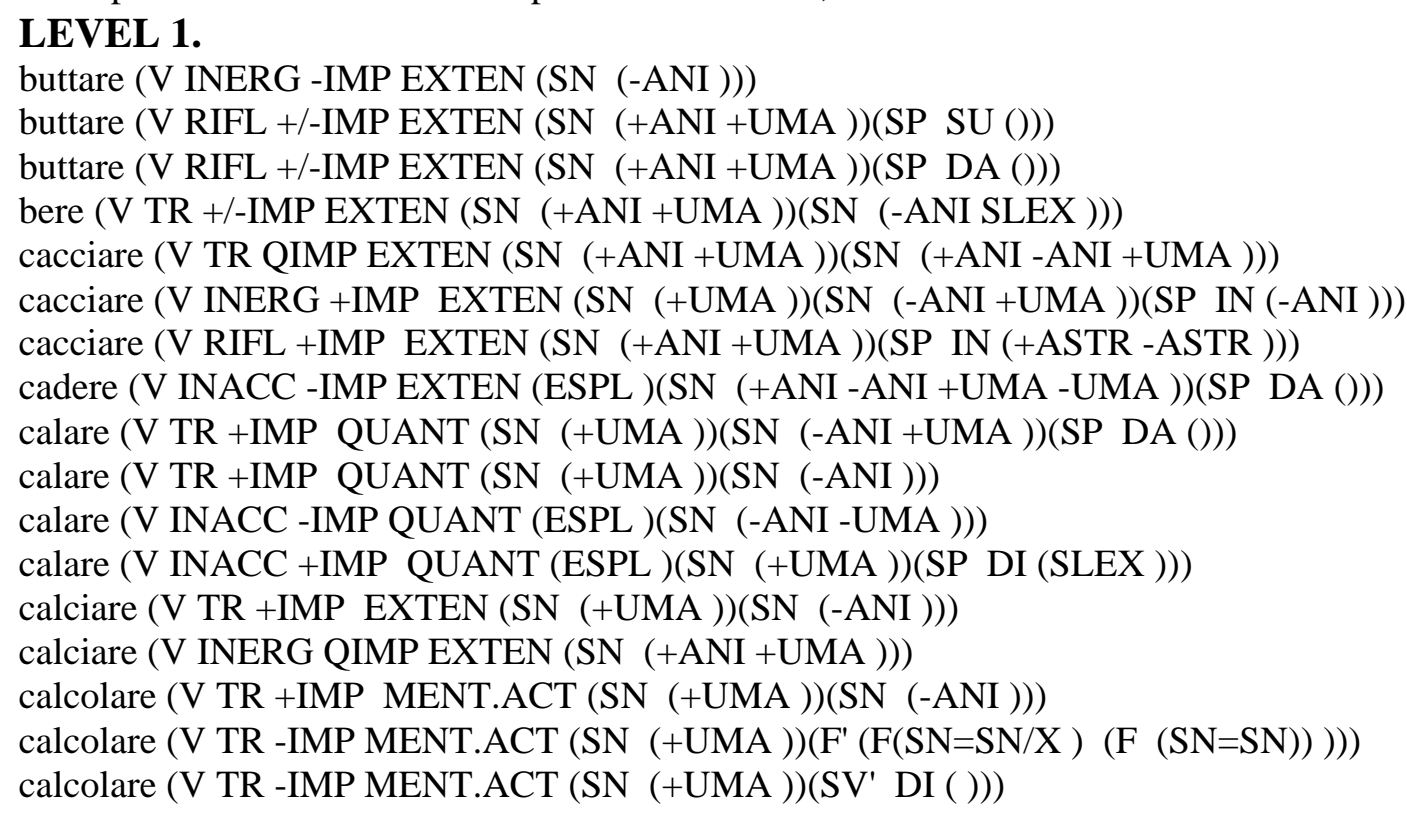


calmare (V TR +/-IMP PROPR (SN (-ANI +UMA ))(SN (-ANI +UMA +ASTR )))

calmare (V RIFL +/-IMP PROPR (SN (+ANI +UMA -UMA +ASTR )))

calpestare (V TR QIMP EXTEN (SN (+ANI +UMA ))(SN (-ANI +ASTR )))

calunniare (V TR +IMP EXTEN (SN (+UMA ))(SN (+UMA )))

calzare (V TR +IMP EXTEN (SN (+UMA ))(SN (-ANI)))

calzare (V INERG -IMP EXTEN (SN (-ANI )))

cambiare (V TR +IMP INCHO (SN (+ANI +UMA ))(SN (-ANI -NUM +UMA +ASTR )))

cambiare (V TR +IMP INCHO (SN (+UMA ))(SN (-ANI -NUM ))(SP IN (-ANI )))

cambiare (V INACC +/-IMP INCHO (ESPL )(SN (-ANI +UMA -UMA )))

cambiare (V RIFL +IMP INCHO (SN (+UMA )) )

cambiare (V RIFL -IMP INCHO (SN (-UMA +ASTR ))(SP IN (-UMA +ASTR )))

camminare (V INERG +/-IMP EXTEN (SN (+ANI -ANI +UMA -UMA )))

\section{Translation Rules from Syntactic Subcategorization into Grammatical Functions}

NP ADV (first or second argument) ---> OBLIQUE

NP (first argument) ----> SUBJECT

NP (first argument) ---> OBJECT (inaccusative verbs)

NP (second argument) ---> OBJECT

PP (second or third argumen + Prep/A) --> INDOBJECT/OBJECT(2)

PP (second or third argument) --> OBLIQUE

Infinitival(first argument) ---> SUBJECT(only adjectives)

Infinitival (second o third argument) ---> VCOMP

PredicativeAP (second or third argument) $\rightarrow$ ACOMP

PredicativeNP (second or third argument) $->$ NCOMP

Finite F (second or third argument) --> FCOMP

Finite F (first or second argument) --> SUBJECT

PredicativePP (second or third argoment) -> PCOMP

PPmodifiers (non-obligatory) ----> OBLIQUE

PPadjuncts (non obligatory) -----> ADJCNT

\section{LEVEL 2.}

buttare (V INERG -IMP EXTEN ( SOGG1 (-ANI )))

buttare (V RIFL +/-IMP EXTEN ( SOGG1 (+ANI +UMA ))( OBL SU ()))

buttare (V RIFL +/-IMP EXTEN ( SOGG1 (+ANI +UMA )) ( OBL DA ()))

bere (V TR +/-IMP EXTEN ( SOGG1 (+ANI +UMA ))( OGG1 (-ANI SLEX )))

cacciare (V TR QIMP EXTEN ( SOGG1 (+ANI +UMA ))( OGG1 (+ANI -ANI +UMA )))

cacciare (V INERG +IMP EXTEN ( SOGG1 (+UMA ))( OGG1 (-ANI +UMA ))( OBL IN (-ANI )))

cacciare (V RIFL +IMP EXTEN ( SOGG1 (+ANI +UMA ))( OBL IN (+ASTR -ASTR )))

cadere (V INACC -IMP EXTEN (EXPL SOGG1)( OGG1=SOGG1 (+ANI -ANI +UMA -UMA ))( OBL DA

()$))$

calare (V TR +IMP QUANT ( SOGG1 (+UMA ))( OGG1 (-ANI +UMA ))( OBL DA ()))

calare (V TR +IMP QUANT ( SOGG1 (+UMA ))( OGG1 (-ANI)))

calare (V INACC -IMP QUANT (EXPL SOGG1)( OGG1=SOGG1 (-ANI -UMA )))

calare (V INACC +IMP QUANT (EXPL SOGG1)( OGG1=SOGG1 (+UMA ))( OBL DI (SLEX )))

calciare (V TR +IMP EXTEN ( SOGG1 (+UMA))( OGG1 (-ANI )))

calciare (V INERG QIMP EXTEN ( SOGG1 (+ANI +UMA ))

calcolare (V TR +IMP MENT.ACT ( SOGG1 (+UMA ))( OGG1 (-ANI )))

calcolare (V TR -IMP MENT.ACT ( SOGG1 (+UMA ))(PROP (FCOMP (SOGG=SOGG1/X)) /EXC

(FCOMP (SOGG=SOGG1)) )))

calcolare (V TR -IMP MENT.ACT ( SOGG1 (+UMA ))(VCOMP DI (SOGG=SOGG1 )))

calmare (V TR +/-IMP PROPR ( SOGG1 (-ANI +UMA ))( OGG1 (-ANI +UMA +ASTR )))

calmare (V RIFL +/-IMP PROPR ( SOGG1 (+ANI +UMA -UMA +ASTR )))

calpestare (V TR QIMP EXTEN ( SOGG1 (+ANI +UMA ))( OGG1 (-ANI +ASTR ))) 
calunniare (V TR +IMP EXTEN ( SOGG1 (+UMA ))( OGG1 (+UMA )))

calzare (V TR +IMP EXTEN ( SOGG1 (+UMA ))( OGG1 (-ANI )))

calzare (V INERG -IMP EXTEN ( SOGG1 (-ANI )))

cambiare (V TR +IMP INCHO ( SOGG1 (+ANI +UMA ))( OGG1 (-ANI -NUM +UMA +ASTR )))

cambiare (V TR +IMP INCHO ( SOGG1 (+UMA ))( OGG1 (-ANI -NUM ))( OBL IN (-ANI )))

cambiare (V INACC +/-IMP INCHO (EXPL SOGG1)( OGG1=SOGG1 (-ANI +UMA -UMA )))

cambiare (V RIFL +IMP INCHO ( SOGG1 (+UMA )))

cambiare (V RIFL -IMP INCHO ( SOGG1 (-UMA +ASTR ))( OBL IN (-UMA +ASTR )))

camminare (V INERG +/-IMP EXTEN ( SOGG1 (+ANI -ANI +UMA -UMA )))

\section{Rules for the transduction from grammatical functions into theta-roles}

1. AGENT, CAUSER, THEME, PATIENT, EXPERIENCER, LOCATIVE, POSSESSOR, CREATOR, APPOSITION, INSTRUMENTAL for SUBJECT

2. THEME, PATIENT, EXPERIENCER, AGENT POSSESSOR for OBJECT1

3. GOAL, THEME, LOCATIVE for INDOBJECT

4. SOURCE, GOAL for $\mathrm{PP}_{\mathrm{da}} / \mathrm{OBLIQUE}$

5. BENEFACTIVE, CAUSER, GOAL, LOCATIVE for $\mathrm{PP}_{\mathrm{per}} / \mathrm{OBLIQUE}$

6. MALEFACTIVE for $\mathrm{PP}_{\text {contro }}$ /OBLIQUE

7. COMITATIVE, INSTRUMENTAL for $\mathrm{PP}_{\mathrm{Con}} / \mathrm{OBLIQUE}$

8. LOCATIVE for $\mathrm{PP}_{\mathrm{Su}} / \mathrm{OBLIQUE}$

9. THEME, SPECIFICATION for $\mathrm{PP}_{\mathrm{di}} /$ OBLIQUE

10. THEME for $\mathrm{PP}_{\mathrm{a}} / \mathrm{OBLIQUE}$

11. LOCATIVE for PP davanti_a $/$ OBLIQUE

12. THEME, for VCOMP

13. PROPOSITION, QUESTION, EXCLAMATIVE for SCOMP

14. EXPLETIVE for []

15. NATTRIBUTIVE for NCOMP

16. AATTRIBUTIVE for ACOMP

17. LOCATIVE for PP intorno_a/OBLIQUE

18. AGENT for $\mathrm{PP}_{\text {da_parte(di) }} / \mathrm{OBLIQUE}$

19. MATTER for $\mathrm{PP}_{\mathrm{di}}$ in $/$ OBLIQUE

20. TEMPORAL for $\mathrm{PP}_{\mathrm{tra}} / \mathrm{OBLIQUE}$

21. TEMPORAL for $\mathrm{PP}_{\mathrm{fra}} / \mathrm{OBLIQUE}$

22. BENEFACTIVE, DIRECTION for $\mathrm{PP}_{\text {verso }} / \mathrm{OBLIQUE}$

23. LOCATIVE for PP in_a $_{-} /$OBLIQUE

Here below we indicate rules for decoding from functional labels into theta-roles:

The translation from functional specifications into thematic roles works according to the following scheme:

- all SUBJECT arguments are translated into AGENT role

- all OBJECT arguments are translated into THEME role

This rule constitutes the default value assigned to Subject and Object arguments. This default rule however is applied after all exceptions have been computed. The exceptions make use of a number of features available in Level 1 representation and can be divided up into syntactic and semantic exceptions to the default rule. We shall review first syntactic exceptions.

1. Exceptions for Subject decoding:

a. Syntactic

- the verb is classified as Psychic or as Inherent Reflexive ---------> THEME

- the verb is classified as Perception verb ----------> EXPERIENCER 
- the verb is classified as Inaccusative

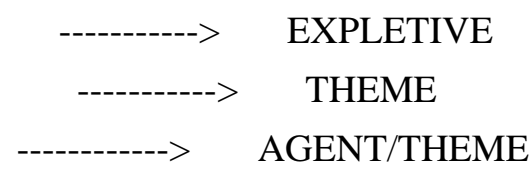

- the noun is classified as Ambiguous POSS/CREAT

- the noun is classified as Ambiguous and is a Pure Nominal

- the noun is classified as Copular

- the noun is classified as a Pure Nominal APPOST

- the adjective has an NP POSS

b. Semantic

- the features associated to the Subject are NOT [+HUMAN] HEAD

- the verb is classified as Inergative and the Aspectual feature is STATIVE CAUSER

- the verb is classified either as Inherent Reflexive or as Psychic and the feature associated to the Subject is [+HUMAN] PATIENT

2. Exceptions for Object decoding

a. Syntactic

- the verb is classified as Inaccusative

THEME

- the verb is classified as Inherent Reflexive or Psychic

EXPERIENCER

- the noun is classified as pure common Noun

AGENT

\section{b. Semantic}

- the verb is classified as Inaccusative and the Aspectual feature is ACTIVITY

- the features associated to the Object are either [+HUM] or [+ANIM] $\rightarrow$ AGENT

The remaining decoding instructions are quite simple and are derived straightforwardly from functional labels. Some difficulties derive from ambiguous prepositions for Oblique argument decoding. In particular, a. CON (with) PP argument can either be computed as COMITATIVE or as INSTRUMENTAL; the default value is INSTRUMENTAL and exceptions are both syntactic and semantic, as follows,

- the verb is Reflexive COMITATIVE

- the feature associated to the PP argument is either [+HUM] or [+ANIM] $>$ COMITATIVE

b. another exception derives from PP with preposition VERSO(towards) which can be computed either as BENEFACTIVE or as DIRECTION; the default value is BENEFACTIVE and the other value is derived as follows,

- the verb is classified as Reflexive

DIRECTION

c. another exception derives from PP with preposition PER(for) which can be computed either as PLACE or as BENEFACTIVE; the default value is BENEFACTIVE and the other value is derived as follows,

- the verb is classified either as Psychic or as Reflexive inherent ----------> PLACE

d. nouns' oblique argument PP with DA(from) preposition is not computed as SOURCE but as GOAL if the noun is a Pure Nominal.

More complex and inherently semantic disambiguation procedures are required for the following classes of noun predicates:

e. nouns' oblique argument PP with DI(of) preposition is computed as SPECIFICATION if the noun is a Pure Nominal and it is not EVENTive;

f. nouns' oblique argument PP with PER(for) preposition is computed as GOAL if the noun is a pure nominal and is neither SUBJECTive nor EVENTive;

g. nouns' oblique argument PP with PER(for) preposition is computed as GOAL if the noun is a reflexive 
and is EVENTive.

\section{LEVEL 3.}

$\operatorname{pred}($ verb,abbagliare,achv,exten,[causer/[+abst],patnt/[+hum,lexs]])

pred(verb,abbagliare, achv,exten,[causer/[+ani,-ani,+hum,-hum],patnt/[+ani,+hum],instr/con/[]])

$\operatorname{pred}($ verb, abbagliare, achv, exten, [causer/[+ani,+hum] ])

$\operatorname{pred}($ verb, abbaiare, activ, exten, [causer/[+ani],theme/a/[]])

pred(verb, abbandonare, achv, exten,[causer/[-hum],patnt/[+ani,+hum]])

$\operatorname{pred}($ verb,abbandonare, achv,exten,[causer/[+ani,+hum],patnt/[+ani,+hum],theme/a/[]])

$\operatorname{pred}($ verb,abbandonare, achv, exten,[causer/[+ani, +hum],theme/[-ani],locat/su/[]])

pred(verb,abbandonare, momnt,exten,[agent/[+hum]])

pred(verb,abbandonare,momnt,exten,[agent/[+hum],goal/a/[+abst]])

pred(verb,abbandonare,momnt,exten,[agent/[+hum],locat/su/[-ani,-abst]])

pred(verb,abbassare,accom,exten,[agent/[+hum],theme/[-ani]])

pred(verb,abbassare,accom,exten,[causer/[-ani,+hum,-hum]])

$\operatorname{pred}($ verb,abbattere,momnt,exten,[causer/[-hum],theme/[-ani,+hum]])

pred(verb,abbattere,activ,exten,[agent/[+hum],patnt/[+ani,-ani,+hum],instr/con/[]])

$\operatorname{pred}($ verb, abbattere,activ,exten,[agent/[+hum]])

$\operatorname{pred}($ verb,abbattere,,statv,exten,[theme/[-ani,-hum],locat/su/[-ani,-abst]])

$\operatorname{pred}($ verb,abbeverare,activ, exten,[agent/[+hum],patnt/[+ani],theme/a/[]])

$\operatorname{pred}($ verb, abbeverare, activ, exten, [causer/[+ani],theme/a/[]])

pred(verb,abbonare,achv,exten,[agent/[+hum],patnt/[+hum],goal/a/[-ani]])

pred(verb,abbonare,,achv, exten,[agent/[+hum],goal/a/[-ani]])

pred(verb,abbondare,statv,quant,[theme/[-ani]])

pred(verb,abbondare,statv,quant,[theme/[-ani],theme/di/[-ani]])

pred(verb,abbondare,,statv,quant,[theme/[+hum,+ani],theme/in/[-ani,+abst]])

$\operatorname{pred}($ verb, abbottonare, accom, exten,[agent/[+hum],theme/[-ani],theme/a/[]])

pred(verb,abbottonare, activ, exten,[agent/[+hum]])

pred(verb,abbottonare,accom,exten,[agent/[+hum],theme/[-ani]])

Here are some of the rules to pass from level 3 into level 4.As appears clearly from the iteration of the same thematic-roles, in order to produce an adequate conceptual representation semantic categories must be used, as well as aspectual categories which are integrated into the representation, together with a modality operator IDENT(identity), CIRC (circumstantial) etc.

(EVENT CAUSE ((THING X) ) EVENT GO-IDENT ((THING <-- ((AGENT X) ) THEME/PATIENT

(EVENT CAUSE (( THING X) (EVENT GO-CIRC ((THING <-- ((AGENT X) ) THEME/PATIENT

(EVENT CAUSE ((THING X) ) (EVENT STAY ((THING <-- ((AGENT X) ) THEME/PATIENT

(EVENT CAUSE ((THING X) ) EVENT STAY-POSS ((THING <-- ((AGENT X) $($ THEME/PATIENT

(EVENT LET $(($ THING X) $)$ EVENT GO $(($ THING <-- $((($ AGENT X) $)((T H E M E / P A T I E N T)$

(EVENT LET $(($ THING X) $)$ EVENT GO-CIRC $(($ THING <-- $((($ AGENT X) $)($ THEME/PATIENT

(EVENT LET $(($ THING <-- $((($ AGENT

(EVENT GO ((THING $(X ' S$ GAZE) $<--\quad(($ EXPERIENCER X

(EVENT STAY ((THING $(X ' S$ GAZE) $<--\quad(($ AGENT X

(EVENT STAY $(($ THING <-- $(($ AGENT

(EVENT STAY-CIRC $(($ THING <-- $(($ AGENT

(STATE BE $(($ THING $<--\quad((T H E M E$

(STATE BE-IDENT ((THING <-- ((THEME

(STATE SHOULD-BE $(($ THING <-- $(((T H E M E$

(STATE BE-POSS ((THING <-- ((THEME/PATIENT

(STATE ORIENT-EMOT ((THING <-- ((EXPERIENCER

(PATH (FROM (THING <-- $\quad(($ SOURCE 
(PATH (FROM-POSS (THING <-- $\quad(($ SOURCE

(PATH (AWAY-FROM $($ THING <-- $(($ SOURCE

(PATH (FROM (PLACE <-- $(($ SOURCE

(PATH (TOWARD (THING <-- $(($ GOAL

(PATH TO-POSS (THING <-- ((GOAL/BENEFACTIVE

(PLACE (IN X'S MIND) <-- (THEME X

(PLACE AT (THING <-- (LOCATION

(PLACE AT-POSS (THING <-- (POSSESSOR

\section{LEVEL 4.}

100. PROPOSE

(EVENT ORIENT-CIRC ((THING X) (PATH TOWARD-CIRC (EVENT (PROP)))))

(EVENT ORIENT-CIRC ((THING X) (PATH TOWARD-CIRC (EVENT (PROP)))))

(EVENT CAUSE ((THING X) (EVENT ORIENT-CIRC ((THING Y) (PATH TOWARD-CIRC (THING

$\mathrm{Z})))))$ )

101. REPORT

(EVENT CAUSE ((THING X) (EVENT GO-CIRC ((REP (S)) ) PATH TO-CIRC (THING (Y)

$($ SOMEONE)) $)))))$

(EVENT CAUSE ((THING X) (EVENT GO-CIRC ((REP (THING Y))

(PATH TO-CIRC (THING (Z) (SOMEONE)))))))

102. REMEMBER

(EVENT GO ((REP (THING X)) (PATH (TO X'S MIND))))

(EVENT GO ((REP (EVENT S)) (PATH (TO X'S MIND))))

(EVENT STAY ((REP (EVENT S)) (PLACE (IN X'S MIND))))

105. WATCH

(EVENT STAY ((THING (X'S GAZE)) (PLACE AT (THING Y))))

(EVENT STAY ((THING (X'S GAZE)) (PLACE AT (EVENT V ((THING Y) (PATH Z))))))

106. LAY

(EVENT CAUSE ((THING X) (EVENT GO ((THING Y) (PATH TO (PLACE ON (THING Z)))))))

107. PERMIT

(EVENT LET ((THING X) (EVENT GO-CIRC ((THING Y) (PATH TO-CIRC (EVENT S))))))

108. KILL

(EVENT CAUSE ((THING X) (EVENT GO-IDENT ((THING Y)

(PATH (FROM-IDENT (PROPERTY ALIVE)) (TO-IDENT (PROPERTY DEAD))))))) [(Y +ANIM)] 109. REMOVE

(EVENT CAUSE ((THING X) (EVENT GO ((THING Y) (PATH (FROM (PLACE (Z)))

$($ TO $($ PLACE $(\mathrm{K})))))))\{($ Z (SOMEWHERE1) $)(\mathrm{K}($ SOMEWHERE2) $)\}$

115. PAINT

(EVENT CAUSE ((THING X) (EVENT GO-EX ((THING Y) (PATH TO-EXISTENCE)))))

(EVENT CAUSE ((THING X) (EVENT GO-IDENT ((THING Y) (PATH TO-IDENT (PROPERTY Z))))) 


\section{The Parser}

Our parser is based on a set of robust Linguistic Modules which take into account lexical forms as well as a syntactic restrictions, and produces two levels of representation in line with LFG theoretical framework: cstructure and f-structure, the former being a tree accessible from syntactic constituents, the latter being a directed graph accessible from attribute-value pairs. Onto this latter structure anaphoric binding of pronominals is computed(see Bianchi, Delmonte, 1989a). The ouput from these modules is connected to an interface between sentence and discourse level constituted by a focus algorithm, which selects on the basis of linguistic criteria what can constitute new information from what is old information and can be introduced anaphorically(see in particular C.Sidner, 1983). The focus algorithm is the necessary premise for anaphora resolution as well for discourse coherence and is conceived as an interface between the syntactic-functional representation of the sentence and the representation of the same sentence at discourse level. Focussing is responsible for anaphora and coreference: i.e. the bringing into attention of a specific set of concepts, facts and actions is a basic mechanism for narrowing down the scope of pronouns, definite and indefinite descriptions, as well as other linguistic means for turning generic into specific descriptions. The scope of quantifiers and quantified NPs is computed on a Logical Form.

The interaction between topic and focus information determines the structure of the message at discourse or text level. The representation constructed by the system is passed to the Inference Module which builds up the appropriate inferences onto the output of the grammar: this is worked out according to lexical-functional grammar a formalism developed theoretically by Bresnan \& Kaplan(in Bresnan, 1982).

The parser computes a level of c-structure where syntactic restrictions are made to apply. This is mapped onto a level of f-structure representation, where all semantic interpretation is worked out. The temporalaspectual parser elaborates on lexical features such as $[ \pm$ EVENTIVE] or $[ \pm$ STATIVE], as well as the presence of temporal adjuncts in order to assess the temporal location of the event referred to by the main verb of the sentence. This is done according to the classical tripartite structure of temporal representation proposed by Reichenbach - speech time, event time, reference time - and the interaction with tense expressed both by the main verb and by temporal adjuncts. The computation of temporal aspectual information cannot be carried out on the basis of the grammatical representation alone, nor without it: this is particularly true seen that optional complements may be realized in the sentence and the aspectual valency may thus have to be updated. Semantic information is then mapped onto conceptual representations which can be regarded as logical forms where operators like quantifiers, negation, modality and tense are made to interact with predicate-argument structures. The following diagram gives a general idea of the linguistic modules:

\section{TABLE I. The General System for Linguistic Computation}

LEXICON:predicate-argument structures---------> CONCEPTUAL REPRESENTATIONS

LFG Grammatical Representation

c-structure ---> f-structure ---> temporal-aspectual computation --->

INFERENTIAL MODULE-----> output

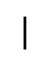

Anaphoric Binding

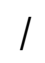

Discourse Reference $<$-.---.---.-- > LOGICAL FORM 
The semantic classes described in the lexicon are reflected in conceptual representations only as far as the main partition between extensional vs intensional class is concerned: this is expressed by the ontological class REP(resentational), which is introduced by Jackendoff(1985a, 1987b) to encode the meaning of intensional verbs, encompassing thus "verbs of opinion", "verbs of belief", as well as verbs encoding in general representations of the world. Another conceptual operator used is TR(ansparent), introduced by Jackendoff in association with "transparent descriptions," and is related to all statements or descriptions involving knowledge of the world by the subject, that is the fact that the situation or event corresponds to reality in the actual world. TR is usually associated with verbs like "know." Conceptual representations have been integrated by tense/aspectual features and this step is crucial in determining inferences, as we shall see.

\section{Interpreting tense}

Natural language tenses are generally treated as sentential operators in semantic theories. What motivates such treatments is the widespread assumption that the tense of a sentence affects the temporal interpretation of all expressions in the sentence. This in turn rests on the assumption that all expressions are interpreted relative to times.

Classical analyses, where tense is treated as a sentential operator, automatically make predictions about the temporal interpretation of NPs, but the evidence provided by Enç 's paper(1986) indicates that the interpretation of NPs is temporally independent of the tense that is present in the syntax. At first glance, a semantic analysis which treats tense as a sentential operator seems to account for certain temporal ambiguities in a simple and elegant way, by appealing to differences in scope relations between NPs and the tense. In the example below (his 1.),

(1) The president was a fool

the reference associated to the NP "the president" is taken to be ambiguous, depending on whether we are talking about a past president or the present president. The ambiguity of (1) is explained by saying that the denotation of the noun "president" will vary according to whether or not it is in the scope of the past tense operator. Thus the tense operator in effect shifts the evaluation time. For example "Mary came" is true when uttered at time $t$ if and only if there is a time $t$ ' prior to $t$ such that Mary come is true at $t$ '. And of course "Mary come" will be true at $t^{\prime}$ just in case the individual denoted by Mary at $t$ ' is a member of the set denoted by "come" at t', i.e. if Mary is included in the set of individuals who came at t'. Assuming that names are rigid designators, the denotation of Mary will be the same at all times, therefore the scope of the tense operator will not affect its interpretation. However, when the head of the NP is a set-denoting non-rigid noun like "president, bird, brain," the scope of tense will be crucial in determining the denotation of the NP. Going back to (1), suppose tense has wide scope over the subject: then the sentence will be true on that reading if and only if there is a past time when "the president be a fool" is true. And this tenseless sentence will be true at that past time if and only if the unique member of the set of presidents at that time is also a member of the set of fools at that time. Thus the tense operator shifts the evaluation time before the subject NP is evaluated, and the denotation of the head noun "president" is obtained by looking at the past time. This gives us the reading where we are saying of an individual who was president at some past time that he was a fool during that same past time.

Suppose we want to assert something about someone who is both a present and a past president simultaneously: there is no way to get this interpretation in that the subject is either within the scope of tense and gets interpreted at a past time, or it is outside the scope of tense and gets interpreted at the present - or 
the Speech Time.

In our system, the computation of tense starts from the tensed main verb and does not affect the SUBJect unless the predicate is actually a state predicate, or as in the example above the sentence is a copular sentence and there is only a predication involved. Broadly speaking, one may say that whenever we have a proposition and an EVENT is involved this implies obligatorily a CHANGE OF STATE in the internal argument of the predicate, the THEME argument. Following Roberts(1986) we define this notion as follows: "some property of the Theme held before the time with respect to which the proposition containing the predicate is evaluated and fails to hold after that time, or viceversa (the property in question can be that of being a certain position, so change of state subsumes change of location). In other words, the truth of positive declarative sentences containing internal Theme arguments entails that the referent of the Theme argument undergoes a change of state"(p.210). Different consequences are predicted for clauses containing predicates belonging to other aspectual classes. In particular, if we take for granted the Dowty/Vendler classification, eventive predicates may include Accomplishments and Achievements, but must exclude Statives and Activities. In the latter cases Themes may appear only as SUBJects and thus be external to the scope of tense. As we shall see, tense can be regarded as an operator binding variables within the predicate: arguments included in the predicate and also adverbials and verb modifiers are all within the scope of the tense operator which will assume a specific or deictic reference according to its lexical nature.

Temporal Dependence is extended to modifiers like adjectivals used in primary and secondary predication and manner adverbs: both categories can be predicated either of the OBJect - thus inducing temporal dependency - or of the SUBJect thus simply describing a property of the SUBJect. Consider the following paradigm:

(2) a. John has become very tall

b. Mary has seen John drunk

c. John ate the meat raw/Jack served the drinks cold

d. Frank considers Tom intelligent

e. Ann left the room sad

f. Sam is bald/Simon seems happy

$\mathrm{g}$ They hammered the metal flat/Tim painted the house red

h John made Mary angry

There is a clear difference in status between the adjectives in $\mathrm{g}$. and $\mathrm{h}$. and those in e. and f.: the former are obligatory or optional complements of a predicate which involves change of state, and an AGENT as a SUBJect; the latter are optional or obligatory complements of predicates which do not involve such a change of state. Thus no temporal dependency may be inferred. The example in d. is a case of lack of temporal dependence owing to the nature of the predicate CONSIDER, a propositional attitude verb which represents the attitude of the SUBJect towards the OBJect NP. In LFG terms, this NP is computed as a non-argument function, interpreted as the SUBJect of the open complement "intelligent" which semantically selects it. The adjectives contained in a. b. c. all denote states: however, "tall" can be regarded a permanent state which can be modified with some difficulty! Raw, cold and drunk on the contrary are temporary states which denote a particular condition associated to the OBJect NP, thus temporally dependent in their interpretation.

As we shall see, to take these differences into account we use an accurate lexical classification, as well an efficient grammatical parser which associates modifiers to the appropriate complement. State-descriptive predicates, or Carlson(1977) stage-level predicates are selected by these XCOMPs: naked, tired, sick, drunk, 
open, insane, crazy, orange, fat, smart in the same way in which Property-predicates, or Individual-level predicates are disallowed from entering an XCOMP in case it is controlled by a temporally dependent OBJect: tall, bald, boring, intelligent.

The same intuitions can be applied to adverbials and locative adjuncts. Consider adverbials discussed by Jackendoff (1972), as belonging to five distributional classes, according to whether they can appear initially in a clause, in auxiliary position, or final:

Class I: can appear in all three positions but with varying meaning (cleverly, carefully,deliberately, voluntarily, intentionally, clumsily...)

Class II: appear in all three positions with no meaning change (quickly, slowly, quietly, frequently...) - also called scopeless adverbials(see...)

Class III: do not appear in final position (evidently, probably, unbelievably...)

Class IV: only appear in auxiliary and final position (completely, easily, totally...)

Class V: final position only (hard, well, more...)

Class VI: auxiliary position only (merely, virtually...)

We assume that adverbs are predicates which do not have arguments nor select theta-roles, but are predicated of a certain grammatical function: class I adverbs are functionally controlled by the SUBJect; class II and III are bound by tense and aspect at clause level; class IV adverbs are temporally dependent and predicate only within the VP, thus they predicate of the result of the action or event expressed by the verb. As Roberts(p.81) proposes, thematic properties are relevant for the selection of some classes: in particular, class I adverbs require an Agent or an Eventive predicate, while class II adverbs only require that the predicate be eventive, as the following example clearly shows (Roberts, 1986, pp.43-46), where we indicate aspectual classes, which are clearly involved in the selection:

ACCOMPLISHMENTS(make,kill,build,paint,cook etc.)

(3) a. *The crystal deliberately dissolved.

b. John deliberately built a house.

c. The crystal quickly dissolved.

d. John quickly built a house.

STATIVES(be asleep,be tall,exist,resemble,believe,etc)

(4) a. *John was deliberately asleep.

b. *John was quickly asleep.

c. John was deliberately polite.

d. *John was quickly polite.

ACTIVITIES(cry,smile,seek,pay attention,roll down,walk,run,etc)

(5) a. *The box deliberately roll down the hill.

b. The box quickly rolled down the hill.

c. John deliberately walked.

d. John quickly walked.

ACHIEVEMENTS(notice,spot,hear,recognize,realize, acquire,receive,lose,etc)

(6) a. *John deliberately noticed the stolen painting.

b. John quickly noticed the stolen painting.

c. ??John deliberately lost/realized it.

d. John quickly realized/lost it. 
Finally, the same arguments can be held to bear on the interpretation of locative PPs: they are either temporally dependent or independent according to whether they are optional arguments of the verb, as the following examples show:

(7) a. John saw the man in the park $\mid--$ The man was in the park

b. John saw/signalled Mary from the kitchen |-- John was in the kitchen

c. John brought/led Mary from the kitchen |-- Mary was in the kitchen

d. John killed/saw/found Mary at the meeting |-- Mary was at the meeting

e. John noticed/mentioned/cursed Mary at the meeting |-- John was at the meeting

f. John fell/leapt from the bus |-- John was in the bus

g. John came/travelled/ran from London to Cambridge |-- John was in London at time t1 and he was in Cambridge at time $\mathrm{t} 2$ and $\mathrm{t} 1>\mathrm{t} 2$.

We offer a final note on the properties of infinitivals or open complements functionally controlled either by the predicate internal argument, an OBJect or an OBJect2, or by the predicate external argument, the SUBJect:

(8) a. John promised to leave $=$ Bill tried to leave

b. John allowed Mary to leave

only in b. can we say that the event is temporally dependent and has a spatio-temporal location in the world.

\subsection{Incorporating tense and aspect and inferential processes}

As various verbs can express either activities or accomplishments in different contexts, several tests have been worked out to separate the former from the latter. Most of them are based on the restrictions on the form of time adverbials that these two classes of verbs can take and are very reliable, so that they make further testing unnecessary. Besides they show that ADJuncts are an essential component of the temporalaspectual representation and of the inferential processes.

(9) a. ?John painted a picture for an hour. / b. John painted a picture in an hour.

(10) a. John walked for an hour. / b. *John walked in an hour.

Even though (9)a and (10)b can be accepted in some contexts, their meaning is very different from that of their more common counterparts. (9)a can in fact be roughly paraphrased as:

(9)a'. John painted at a picture for an hour.

and means that John did not paint a whole picture, while (9)b means that John did paint the picture. Analogously, (10)a implies that John had a walk, while (10)b could only mean that it took him an hour to start walking.

(11) a. It took John an hour to paint a picture (accomplishment). / b. ?It took John an hour to walk (activity).

This test brings to the light a peculiarity of accomplishments, since stative verbs cannot appear in this construction and achievements are interpreted semantically like activities:

(11) c. *It took John an hour to know the answer. / d. It took John an hour to notice the painting.

(12) a. John was walking when he was interrupted.

implies: (12) b. John walked; while from:

(12) c. John was painting a picture when he was interrupted.

it is inferred that: (12) d. John did not paint a picture.

As achievements capture either the inception or the climax of an event, they are represented by language as having virtually no duration. This is the basic concept that underlies the tests for achievement verbs: 
(13)a. John noticed the painting in ten minutes.

entails: (13)b. Ten minutes elapsed before John noticed the painting, rather than:

(13)c. *John was noticing the painting during ten minutes.

As can be noticed by comparing the sentence in (13)b with (10)b, achievements behave like activities with respect to adverbial prepositional phrases with "in." A variation in the definiteness of the subject or object $\mathrm{NP}$, however, makes achievements with for-phrases perfectly acceptable:

(14) a. *John discovered that quaint little village for years.

b. John discovered quaint little villages for years.

c. Tourists discovered that quaint little village for years.

A plural subject or object in fact ensure the iterative reading that makes such sentences semantically acceptable. In sentences (14)a-c, it is part of the meaning of the verb that one cannot discover the same thing more than once, so either a plural subject or a plural object are necessary to express various "discoveringevents." As the temporal adverbial is indefinite, the number of discoveries involved must be indefinite as well.

To summarize(but see also Furlan,1989;Roberts, 1986 p.208; Enç ,1986):

\section{Accomplishments:}

a. Specification of the "moment-when" is OK;

b. "V for an hour" does not entail "V for all moments in that hour;"

c. Progressive does not entail (resultative) perfect;

d. Occurs in complements of process(aspectual) verbs (stop,start,finish,continue,ect);

e. Occurs in complement of "complete."

\section{Statives:}

a. do not occur in the progressive;

b. do not occur with Class I adverbs;

c. do not occur with iterative simple present;

d. allow "simultaneous reading" of the event contained in the main and the state contained in the embedded sentential complement (cfr. a.John said Mary left; where the time of leaving must precede the time of saying - this is called the "shifted reading" by Enç (1986), vs. b.John said Mary knew the answer; where both the "shifted reading" and the "simultaneous" reading is allowed);

e. are temporally independent; in particular, they do not have to be included in the interval denoted by temporal Adverbs (cfr. John visited Italy last year: where the interval referred to cannot be located

before or after the time indicated by the temporal adverb, vs. John know the answer last year, where we want to say that John still knows the answer now).

Activities: a. Specification of "moment when" is bad;

b. "V for an hour" entails "V at all times in that hour"

c. Progressive entails (resultative) perfect;

d. Occurs in complement of process Verbs;

e. Does not occur in complement of "complete";

Achievements: a. Does not occur with specification of "how long";

b. Odd with progressive;

c. Does not occur in complement of process Verbs; 
d. Does not occur in complement of "complete".

e. Allows iterative reading with plural naked NP OBJect/SUBJect and "for" adverbials

\section{Representing Scope in Logical Form}

In the model presented in Table I. we have the following levels of grammatical representation:

(15) a. L-structure: lexical forms and lexical binding

b. C-structure: with functional annotations: syntactic binding and quantifier markers raising

c. F-structure: anaphoric binding

d. P(redicate)-A(rgument) structure: where reconstruction takes place

e. LF: the level that determines interpretation by the rule of Scope Assignment

Logical Form is produced as a multilayered structure in which each argument is assigned a layer. Input to LF is Predicate Argument Structure which is the list of arguments a predicate selects in the lexicon, plus all the information added by the grammar.

LF representation in LFG comes naturally and obligatorily after the other levels of representation: thus, after wh- movement has applied, at c-structure level, and also after anaphoric binding has applied, at f-structure level. Whereas in other theoretical framework it is an open question where to apply certain principles(see Haï k,1984; Higginbotham, 1980,1983,1985; May,1985, 1989), in LFG syntactic variables are only visible at c-structure level, and morphologic-ally expressed or unexpressed anaphoric elements are only visible at $\mathrm{f}$ structure level. Thus, there is no way to make quantifier raising precede long distance dependencies. Since quantifiers may assume scope according to the syntactic structure they are in, and linear precedence also constitutes a relevant criterion for scope assignment, in order to take advantage of syntactic restrictions to quantifier raising, I propose to mark the path a certain quantifier will follow already in the syntax, at cstructure level by introducing empty variables that are raised and become placeholders for quantifiers in the following representations. The criteria are as follows:

\section{A. QM Raising principles(at c-structure)}

1. Each $f$-structure constitutes a referential domain $(R D)$;

2. All quantifiers raise a $Q M$ within their RDs;

3. All QMs are bound in their RDs;

4. Extensions of the RD are allowed( $a$ in a language that encodes modality at $c$-structure( $b$ to the $f$ commanding one if either domain is transparent))

5. An R-domain is a root domain specified for referential energy:

$i$. it is strong if it is referentially transparent

ii. it is weak if it is referentially opaque.

iii. Referential energy :

a. TENSE $=[ \pm R E F], M O D E[ \pm R E A L], C L A S S[ \pm I M P L I C]$, ASPECT $[ \pm P E R F]$;

b. $C A R D=[ \pm D E F / \emptyset], I N D I V[ \pm S P E C]$.

c. transparency obtains whenever the features have positive value.

Our proposal capitalizes on Halvorsen's(1983) use of Placeholders to mark off the presence of NPs with quantifiers in Spec: then, we shall raise a Quantifier Marker, or an empty operator lacking syntactic content but appearing as an atomic attribute-value pair, such as,

(16) $\mathrm{QM}=O_{125}$ 
$\mathrm{PM}=e_{125}$

at clause level, whenever there is a placeholder which contains a variable. The QM bears the same index as the variable which is then associated to the NP by percolation, so that the NP, which contains the restriction will also be appropriately coindexed. On a par with VPs, NPs may then let their features percolate upward to S level(see Zaenen,1983). Semantic features such as Mode, Tense, Aspect, and Semantic Class will affect the interpretation of the clause and the Events; on the contrary, the presence of a quantifier in a NP will only affect the interpretation of the NP where it binds a variable.

The operators binding variables in NPs are justified because no lexical element can be raised in LFG, only empty abstract operators. Besides, a structure Operator...variable is independently motivated in c-structure by syntactic binding: this could be a case of syntactic binding with an empty operator. Here below we show how the steps in order to reach LF are justified by natural language phenomena:

\section{To motivate reconstruction before scope assignment, after wh-movement}

(18) What did everyone buy for Max?

(19) Who bought everything for Max? $\neq$ Who buys everything for Max?

In these example we see that wh- elements must be reconstructed in their position before they can access LF: the result will be the well-known subj-object asymmetries in scope assignment. In particular a universal quantifier in object position will preferentially assume lower scope, i.e. will be given a collective reading in presence of a wh- element in subject position; the opposite applies when the universal quantifier is a SUBJect. This is encoded in pas. and is interpreted in LF because OBJects are assigned scope in the restriction of tense, in case tense has a specific reference. Note the difference in interpretation in (19): if we introduce present tense the preferred scope assignment will produce a distributional reading for "everything." It is clear that quantifiers belonging to a separate clause from the one in which a wh- element is reconstructed do not interact with it, as shown in the following examples:

(20) Which men did someone say that Mary likes t?

(21) Which men did Mary say that some woman loved?

\section{To motivate reconstruction after pronominal binding at $f$-structure}

As a further argument, Williams(1986) notes that only wh- variables can license parasitic gaps: quantifier variables cannot,

(23) a. *John talked to everybody about $\mathrm{t}$

b. Who did John talk to $t$ about $\mathrm{pg}$ ? $\neq *$ A chi Gino ha parlato $\mathrm{t}$ di $\mathrm{pg}$ ?

(24)a. ?Manzoni è uno scrittore che tutti quelli che hanno letto $p g$ ricordano $e /$ Manzoni is a writer that all those who read $p g$ remember $e$

b. *Manzoni è uno scrittore che gli studenti che hanno letto $p g$ ricordano $e /$ Manzoni is a writer that the students who read $p g$ remember $e$

c. ??Manzoni è uno scrittore che gli studenti che $l^{\prime}$ hanno letto ricordano $e$ volentieri/Manzoni is a writer that the students who read it remember $e$ willingly

d. Manzoni è uno scrittore che tutti gli studenti che $\quad l$ hanno letto ricordano $e$ volentieri/Manzoni is a writer that all the students who read it remember $e$ willingly

Parasitic gaps in LFG are computed at f-structure. They can be regarded as "empty clitics" contained in ADJuncts, which are not internally visible at c-structure level: i.e. no syntactic principle can build a path leading from a syntactic binder up in the structure, like a TOPic or a FOCus, down to its bindee, a clitic or an empty element, if this is contained in an ADJunct. In other words ADJunct are syntactic islands but can be made visible at f-structure in LFG. However, consider (23)b. where a parasitic gap is contained in a non- 
clausal OBLique coargument: this is only allowed in English where preposition stranding constitutes a typological option(see Delmonte,1987c). Italian allows no preposition stranding and requires a clausal structure to contain the parasitic gap. Moreover, as shown in (24)a,b,c, it is very restrictive as to the possible containing structures. In particular, when included in a Complex NP(CNP), licensing conditions for parasitic gaps include the referential status of the head, which in this case must be opaque - quantified, and not transparent or definite.

In the following examples we see the relevance of the referential power of quantified NPs and quantifiers, which in some cases licenses pronominal binding but in some other cases forbids it. In particular English personal pronouns can be bound by a quantifier if it precedes or is superior in the structure to it:

(25) a. *He thinks that John is sick

b. *He thinks that everyone is sick

These sentences are ungrammatical but the corresponding one in the right configuration are fine,

(26) Everyone thinks he is sick = Ognuno pensa PRO di essere malato $\neq$ ??Ognuno pensa che pro/egli è malato

(27) Everyone's mother liked him $\neq$ La madre di ognuno ama lui / La madre di ognuno lo ama

The same does not apply for Italian personal pronouns and clitics, as is shown by the translations, which we included every time there is some contrastive difference between the two languages. An opposite behaviour is also witnessed by anaphors like lexical reflexives and possessives, which can be bound within the same clause in English but not in Italian:

(28) a. Himself I think John likes t

b. Which picture of John did he see $\mathrm{t} \neq *$ Quali foto di Gianni ha visto?

c. *Him I think John likes t

(29) a. his father hates John [*Each other's wives murdered the men]

b.* his father hates everyone

c. his father saw John $\neq *$ He saw John $\neq$ Suo padre ha visto Gino

The only case in which a possessive pronoun can be bound in the same clause, is constituted by the longdistance anaphor "proprio" which can be bound by a quantifier in the same clause from OBJect position when a psychic verb is the main predicate. In this sense, "proprio" behaves like "each other" in picture NPs (30)a. Ciascuno è preoccupato della propria salute (Each person is worried about self health)

b. La propria salute preoccupa tutti/molti (Self health worries all/many)

c. Each other's pictures worried Peter and Mary / Pictures of himself upset Tom

Principles operating at LF would then be preceded by the following generalized criterion which creates a quantified structure, each time a QM binds a variable, or a PM(Placeholder Marker) in a NP, in the level of representation which precedes LF, i.e. PAstructure.

\section{B. Reconstruction criteria}

1. All functions containing a FORM (i.e., deprived of PRED(semantic content)) are discarded from the representation;

2. All A'-bound functions (lexical and syntactic variables) are superseded by the binder's attribute;

3. All argument functions are erased in favour of theta-roles.

In this way no empty elements, in particular variables, nor lexical expletives will be left in LF. Only grammatical relations carrying the non-argument discourse functions FOCUS/TOPIC which have pragmatic 
and interpretative relevance will be left in the representation - the same applies to the output of lexical redundancy rules which assign FOCUS to inverted constituents associated to expletives(see Bresnan \& Kanerva, 1989). As a result only the following generalized rules will be maintained in LF.

\section{Scope assignment rule}

\section{All arguments are quantified according to their inherent power;}

\section{No vacuous quantification is allowed}

Since we surmise that quantifier raising applies where syntactic binding also applies, we must at first see what is the structure we are starting from and what is the one we shall have to produce at LF level for scope assignment. A quantification structure to which scope has been assigned in a sentence like (31) is (31)i.:

(31) John saw every car.

(31)i. [Every car $]_{i}[\text { John saw ti }]_{S}$

where we might decompose the effect of scope assignment following Williams(1986, p.266),

(32) a. a quantifier - the determiner of an NP in an $\mathrm{A}^{\prime}$-position is the quantifier

b. a restriction on the range of the variable - the N' of the NP is the restriction

c. a variable - the trace bound by the NP is the variable

d. a scope - the $\mathrm{S}$ in construction with the NP is the scope

And this is his comment: "... in (31)i. the quantifier is every, the restriction is car, the variable is the position occupied by $t$, and the scope is $S$." A strong argument in favour of LF as a separate level of representation is constituted by sentences like (33) below, as also Halvorsen(1983) - though only with respect to his "semantic structures":

(33) Every woman loves some man.

There is no syntactic motivation for more than one f-structure for this sentence, but since the sentence is twoways ambiguous there must be two separate logical forms and two interpretations. Another strong argument comes from quantifiers which must assume broad or wide scope. For example, "each" is a special quantifier which must distribute over something for the sentence to be grammatical, as Williams(1986) notes in his paper.

(34) Who did each boy dance with t?

where each takes wide scope with respect to wh-, being the SUBJect, but also if it is the OBJect,

(35) Who t danced with each boy?

Also note that a sentence is unacceptable whenever each does not have another set available to distribute on,

(36) a. ?? Each patient left. vs All the patients left.

b. Each patient saw his own chart.

Finally, it is possible to raise indefinite NPs out of CNP, which is an island for syntactic variables. However QM raising is subject to one basic condition: the head NP of the predicative adjunct cannot be a quantified NP, as also Halvorsen(1988) notes in the example,

(37) John dates every woman who loves a fish.

where "a fish" cannot assume broad scope and is in the scope of the universal quantifier. A second condition is again related to the subject-object asymmetry: subject indefinite NPs escape more easily from CNP than object ones, as shown by,

(38) a. John dates every student that a colleague recommends.

b. John dates/dated every student that a colleague recommended. 
where we do not want the interpretation in which there is one colleague for each student dated by John. Tense is also relevant: (38)b. is a clear case of the specificity inheritance process for an indefinite NP triggered by the presence of referentiality at clause level, induce by past tense in the verb inflection. The same facts are noted by Chomsky (1977, p.116) for a quantifier like "anyone" which is blocked from assuming scope when the head NP of a CNP in which it is contained is definite,

(39) a. we can't find books that have any missing pages

b. *we can't find the books that have any missing pages

c. *we can't find certain books that have any missing pages

As Chomsky comments(ibid., p.117) "one may interpret 10a. with wide scope for any, as for example, in despair after a search for certain missing pages has failed, even though any is within a complex NP."

\subsection{Running the scope algorithm}

We might characterize the conjoined operation of quantifier raising and scope assignment using Ioup's(1975, p.45) definition of the scope relations intervening between two quanfied expressions, Q1 and Q2:

"Semantically, 'Q1 has higher scope than Q2' means that the remainder of the sentence dominated by the S node that immediately dominates Q1 - and contains Q2 will be interpreted as an instance for every member of the set designated by Q1. As an example, consider the following sentence:

(21) All the women built a garage

If "all the women" is given higher scope, then the predicate "built a garage" applies to each of them in the interpretation and there can be as many garages as there are women. This interpretation, which assigns a Qpl higher scope can be referred to as an INDIVIDUAL interpretation. If, on the other hand, the quantified NP "all the women" is read with lower scope than "a garage," then it will be contained in the predicate that is interpreted for each member of the set "a garage" - in this case just one member - which now has the higher scope. Each time the predicate applies, the set of women is referred to as a group... in this reading there will be just one instance of the predicate, for we have a single garage. Thus the women work collectively to build it. This interpretation, where the indefinite NP Qsg is given highest scope, is referred to as a COLLECTIVE one."

We implemented Hobbs \& Shieber's(1987) basic algorithm plus a number of additions as follows:

1. to account for scope preference, we added a table lookup with a subdivision of quantifiers into classes according to their relative strength: in case two quantifiers are contained in the same clause and belong to the same class, so they can be freely interchanged;

2. alongside opaque operators, we added an Island Operator which prevents quantifiers belonging to separate RDs from interacting (inside or outside the island): the function "apply-opaque-args" controls whether an operator is an island and generates a complete scope for its argument - [ \pm REAL] sentential complements, restrictive relative, SCOMPs to factive and intensional predicates, etc.;

3. pragmatic and discourse functions like TOPIC and FOCUS impose restrictions on the scope of their constituents, so "pull-opaque-args" controls whether a more specific procedure is called for before generating the scope of an opaque argument(negation, for instance).

The algorithm is implemented in Quintus Prolog and runs under VMS on a Microvax-II. Here below are some examples:

i. Gino non è arrivato ieri./John has not arrived yesterday

predicate-argument structure

not(arrivato(Gino< definite : $\mathrm{g}$ :ind $(\mathrm{g})>,<$ event $: \mathrm{e}:$ and(arrivare $(\mathrm{e}),<$ past $: \mathrm{t}:$ and $($ tense $(\mathrm{t}),<$ definite $: \mathrm{i}$

:ieri(i) $>)>)>)$ )

logical form

definite $(\mathrm{g}$,ind $(\mathrm{g})$, 


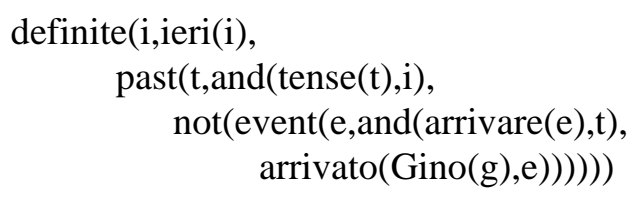

The interpretation should read out something like: there is an individual in discourse, "Gino," and a spatiotemporal location , "yesterday," in the past tense, and there is no event of "arrivare," in the past tense, such that "John arrived" is true. On the computation of tense in logical form see Hinrichs,1988;Hornstein,1989; ter Meulen,1983.

In the following example there is an implicit argument which is turned into a generic existential quantifier deprived of a restriction, and its scope is lower than any other quantified expression of the same sentence, meaning that no individual can be individuated in the previous discourse even though there must be someone who performed the action.

ii. Non è stato promosso Gino ieri./Has not been passed John yesterday(John has not been passed yesterday) $\operatorname{not}(\operatorname{promosso}(<$ exist : $\mathrm{x}: \operatorname{pro}(\mathrm{x})>, \operatorname{Gino}(<$ definite $: \mathrm{g}: \operatorname{ind}(\mathrm{g})>),<$ event $: \mathrm{e}: \operatorname{and}(\operatorname{promuovere}(\mathrm{e}),<$ past $: \mathrm{t}$ :and(tense $(\mathrm{t}),<$ definite $: \mathrm{i}:$ :ieri(i) $>)>)>)$ ) definite(i,ieri(i),

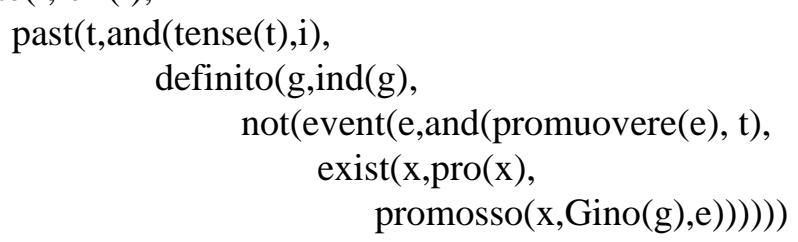

In the following examples "FOCus," the non-argument function inherited from the grammatical f-structure representation is introduced in pas as an "island" operator, in case it contains a quantifier interacting with another quantifier within the same proposition. Subject-object asymmetries are obtained from the presence of temporal-aspectual operators:

iii. Chi ha comprato tutti i regali ieri?/Who bought all the presents yesterday?

comprato(focus $(<$ wh : $\mathrm{k}: \operatorname{chi}(\mathrm{k})>),<$ tutti $: \mathrm{r}: \operatorname{regali}(\mathrm{r})>,<$ event $: \mathrm{e}: \operatorname{and}(\operatorname{comprare}(\mathrm{e}),<$ past $: \mathrm{t}:$ and $($ tense $(\mathrm{t}),<$ definite : y :ieri $(\mathrm{y})>)>)>)$ )

definite(y,ieri(y),

past $(\mathrm{t}$, and $($ tense $(\mathrm{t}), \mathrm{y})$,

$\mathrm{wh}(\mathrm{k}, \operatorname{chi}(\mathrm{k})$,

event (e, and(comprare(e),t),

tutti(r,regali(r),

ha_comprato(focus(k),r,e))))))

iv. Che cosa hanno comprato tutti gli invitati ieri?/What have all the invited people bought yesterday?

comprato(< tutti : i :invitati(i) $>$, focus $(<w h: k$ :che_cosa $(\mathrm{k})\rangle),<$ event $:$ e $:$ and $(\operatorname{comprare}(\mathrm{e}),<$ past $: \mathrm{t}$

:and(tense $(\mathrm{t}),<$ definite : $\mathrm{y}:$ ieri( $(\mathrm{y})>)>)>$ )

definite(y,ieri(y),

past $(\mathrm{t}$, and $($ tense $(\mathrm{t}), \mathrm{y})$,

tutti(i,invitati(i),

event(e,and(comprare(e),t),

ch(k,che_cosa $(\mathrm{k})$, comprato(i,focus $(\mathrm{k}), \mathrm{e})))))$ )

The need to use c-structure in order to assign scope following syntactic restrictions can be derived from the behaviour of "nessuno"(nobody) and of the deictic NP "quello studente" (that student) in the following sentences: being contained in a clause embedded under the matrix predicate "desiderare"(want, wish) which induce a referentially opaque context, also witnessed by the use of subjunctive mode in the embedded clause - differently, then, from what happens with sentential complements of verbs of "saying" which induce a 
transparent context and require the use of the indicative mode in the embedded clause - the two quantified expressions must be raised out of their clause in order to assume scope in the higher matrix.

ix. Il professore non desidera che venga promosso nessuno studente/The professor does not wish that be passed no student

$\operatorname{not}(\operatorname{desidera}(<\operatorname{definito}: \mathrm{p}: \operatorname{professore}(\mathrm{p})>\operatorname{prop}(\operatorname{promosso}(<\operatorname{nessuno}: \mathrm{s}: \operatorname{studente}(\mathrm{s})\rangle,<$ event $: \mathrm{e}$ :promuovere $(\mathrm{e})>))))$

definite(p,professore(p), $\operatorname{not}($ event(e,promuovere(e), nessuno(s,studente(s), desidera(p,prop(promosso(s,e))))))

x. Ogni professore ha incontrato tutti gli studenti che hanno passato l'esame/Each professor has met all the students who have passed the examination

incontrato( $<$ ogni : p :professore $(\mathrm{p})>,<$ tutti : $\mathrm{s}$ :and(studenti(s), prop(isola(passato(s, $<$ definito : e : esame(e) $>,\langle$ event $: \mathrm{p}:$ and $(\operatorname{promuovere}(\mathrm{p}),\langle$ past $: \mathrm{t}: \operatorname{tense}(\mathrm{t})\rangle)>))))>$ )

definite(e,esame(e), ogni(p,professore(p), tutti(s,and(studenti(s), passato(s,e)), $\operatorname{prop}(\operatorname{past}(\mathrm{t}, \mathrm{tense}(\mathrm{t})$, event(p,and(promuovere $(\mathrm{p}), \mathrm{t}))$, incontrato $(\mathrm{p}, \mathrm{s}))))$

\section{Inferences in Conceptual Representations}

Inferences are computed after Logical Form and in a Knowledge Representation language KL-ONE recast in C-Prolog, on conceptual representations which encode lexical static information: the output of the grammar provides information as to tense, adjuncts and modification. Tense is at first made to interact with aspect, optional complements, adjuncts in a parser that assigns interpretation in terms of the three parameters introduced by Reichenbach: TR(reference time), TD(discourse time), TE/TS(event time/state time), mapping the relations intervening between tense in the matrix as expressed by the inflected verb and tense of the remaining arguments of the predicate (for a comprehensive analysis of the mapping see Sanson,1990). We shall show its work by an example:

Ex01. John runs.

pred: run

aspect: activity

mode: finite

tense: present

number: sing

subj : type : +human

head : john

number: sing

det : + def

role : experiencer_agent

aspect : activity

?- compute (ex01,X,Y,Z,T)

$\mathrm{td}=\mathrm{tr} \quad$ included(tr,tes)

matrix time reference $=+$ specific

$\mathrm{X}=\mathrm{td}=\mathrm{tr}$,

$\mathrm{Y}=$ included(tr,tes);

$\mathrm{td}=\operatorname{tr}$ immediately_precedes(tr,tes)

$\mathrm{X}=\mathrm{td}=\mathrm{tr}$

$\mathrm{Y}=$ immediately_precedes(tr,tes) 
different(td,tr) simultaneous(tr,tes)

historical present improbable

$\mathrm{X}=\operatorname{different}(\mathrm{td}, \mathrm{tr})$

$\mathrm{Y}=$ simultaneous(tr,tes)

Ex11: John coughed for three hours

pred : cough

aspect : iterative_achievement

mode: finite

tense : past

number : sing

subj : type : +human

head : john

number : sing

det : + def

role : experiencer_agent

adjunct : pred : for

mod : three

type : duration

aspect : non_punctual

object: type : temporal

pred : hour

num : plur

aspect : iterative_achievement

?- compute(ex11,X,Y,Z,T)

adverb : head : for

type : duration

duration : non_punctual

$\operatorname{td}=\operatorname{tr}$ tes $<\operatorname{tr}$

duration(tr, (hours(three))

$\operatorname{td}=\operatorname{tr} \quad$ tes $<$ tr

matrix time reference: + specific

multiple occurence of the event

$\mathrm{X}=\mathrm{td}=\mathrm{tr}$,

$\mathrm{Y}=$ tes $<\mathrm{tr}$;

This computation is passed on to the inferential engine which has inferential rules associated to conceptual representation templates, mainly derived from Jackendoff, 1976 and subsequent works. The inferential procedure has a set of basic rules which decompose conceptual representations on more elementary predicates like $\mathrm{BE}$, as follows:

a) $[\operatorname{CAUSE}(\mathrm{X}, \mathrm{E})]=>\mathrm{E}$

b) $[$ STAY $([X],[$ AT Y] $)$ from $\mathrm{t} 1$ to $\mathrm{t} 2]=>[\mathrm{BE}([\mathrm{X}],[\mathrm{AT} \mathrm{Y}])$ at $\mathrm{t} 3]$ cond $=\mathrm{t} 1<\mathrm{t} 3<\mathrm{t} 2$

c) $[\mathrm{GO}([\mathrm{X}],[\mathrm{FROM} \mathrm{Y}],[\mathrm{TO} \mathrm{Z}])$ at $\mathrm{t} 1]=>[\mathrm{BE}([\mathrm{X}],[\mathrm{AT} \mathrm{Y}])$ at $\mathrm{t} 2] \&[\mathrm{BE}([\mathrm{X}],[\mathrm{AT} \mathrm{Z}])$ at $\mathrm{t} 3]$ cond $=\mathrm{t} 2<\mathrm{t} 1<\mathrm{t} 3$

d) $[$ GO ([X],[(AWAY_)FROM Y], [TO(WARD) Z]) from t1,to t2] $\Rightarrow$ NOT [STAY ([X],[AT Y] from t1,to

t2] \& NOT [STAY ([X],[AT Z] from t1,to t2

e) STAY ([X],[AT Y]), from t1, to t2] => NOT [GO ([X],[(AWAY_)FROM Y],[TO(WARD) W]) from t3,to t4]

cond $=\mathrm{t} 1<\mathrm{t} 3<\mathrm{t} 4<\mathrm{t} 2$

The readings of the expressions are quite intuitive: in a) if an agent causes $E$ then $E$ takes place; $b$ ) is the subinterval condition which is cast into J.Allen $(1983,1984)$ formalism for time reference computation; c) shows how a motion predicate is translated into a couple of state predicates and so on. Here below are some excerpts from the implementation into KL-ONE in C-Prolog.

i. $\mathrm{BE}(\mathrm{x}, \mathrm{event})$

infer(be(X,Y,T,M,_,Bex),true):-

fact(be(X,Y,T,M,_,Bex),_),

proof(be(X,Bex,T,circumstantial,_,_),true). 


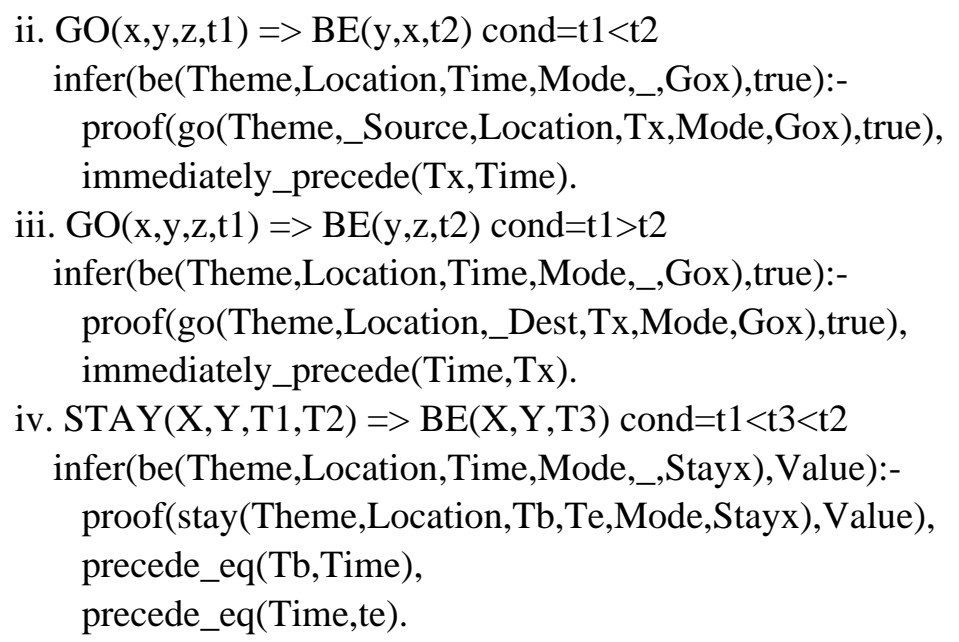

This information is coupled with the conceptual representation associated to each main predicate, with variables added for tense. The output of the inferential engine is a truth value associated to a query about the spatio-temporal location of individuals and events in the world: both predicates and individuals may constitute the query. The following is the listing associated to the sentence "John runs home":

ex03 :- assert(run(john,nil,home,t3,f03),-specific)/X,

run(Theme,Source,Destination,Time,position,Symb,X).

> individual_concept(f03_go)

$>$ individuates(f03_go,go)

>> has_role(f03_go,theme)

assumption : >> has_role(f03_go,theme) $<<$

> satisfies(theme,f03_go,r_theme,go)

$>$ has_role(f03_go,source)

assumption : > has_role(f03_go,source) $<<$

> satisfies(source,f03_go,r_source,go)

>> has_role(f03_go,destination)

assumption : >> has_role(f03_go,destination) $<<$

> satisfies(destination,f03_go,r_destination,go)

>> has_role(f03_go,time)

assumption : >> has_role(f03_go,time) $<<$

>> satisfies(time,f03_go,r_time,go)

> has_role(f03_go,mode)

assumption : >> has_role(f03_go,mode) $<<$

$>$ satisfies(mode,f03_go,r_mode,go)

>> has_value(theme,f03_go,john)

>> has_value(source,f03_go,nil)

>> has_value(destination,f03_go,home)

>> has_value(time,f03_go,t3)

>> has_value(mode,f03_go,position)

end

be_at (john,A,B,C) ?

be_at (John,nil,t2,f03)/true

be_at(John,not nil,t2,f03)/false

In this case, nothing can be asserted of John in relation to his destination: we only know that he was at some place, his source(nil), when he started running. In case we introduce a specific time reference, as in "John went home", things will change abruptly:

ex03bis :- assert(go(john,nil,home,t3,f03bis),+specific)/X,

run(Theme,Source,Destination,Time,position,Symb,X).

the same computation is carried out by the inferential engine, apart from the use of a different index f03bis 
for f03, and clearly a different kind of inference on the destination as shown by the replies to the query:

be_at (john,A,B,C)?

be_at(John,home,t4,f03bis $) /$ true

be_at(John,nil,t2,f03bis $) /$ true

be_at(John,not home,t4,f03bis)/false

be_at(John,not nil,t2,f03bis)/false

\section{Focus and Topic}

A final issue related only partly to the structure of lexical information is relevance as a property associated in general with the subject of predication, or with the content itself of predication. Information expressed by subordinate clauses, definite noun phrases, factive verbs, and non-restrictive relative clauses are marked as being of low relevance. Cleft constructions and the placement of sentence stress usually serve to separate relevant from non-relevant information. Information which is low in relevance should behave under denial and questioning the way presuppositions are observed to behave: such information is preserved because the denial or questioning of information low in relevance would amount to a denial of the relevance of the whole utterance. They suggest that for any sentence a set of grammatically specified entailments may be generated by variable substitution of each syntactic constituent which contains the sentence stress.

Usually, what is expressed as subject of a predication is a TOPIC new or old, and has its own status in the discourse; whereas, by default, what is FOCUS is contained in the predication(see Delmonte, 1985;1987a,1987b;1988a). A number of factors affect this default organization of utterances, most of which are shown either by the syntactic structure and/or by the phonology: like heavy emphatic stress on some constituent. The lexicon can, however, be used to introduce default facts about this bipartition. New TOPICS must be associated with a particular determiner, and pronouns or epithets do not constitute in themselves independent referring expressions and as such can only be treated as old TOPIC. Lexical entries do not in themselves mark TOPIC/FOCUS, but lexical redundancy rules do. In fact there is more than one case in which TOPIC information is automatically associated with functional assignment: subject inversion in locative contexts (see Bresnan \& Kanerva, 1989) is one such case. FOCUS/TOPIC labels are attached to NPs according to grammatical functions and other lexical information at sentence level and they play an important role in the global analysis of a text. However, the partition TOPIC(Subject NP) and FOCUS(rest of the proposition) shows clearly that when a New Topic is introduced, usually with a proper noun or just a common noun, it binds a coindexed anaphoric pronoun in the FOCUS; whereas, when an Old Topic is introduced, usually with a pronominal, it binds a coindexed variable. Identificational sentences when accompanied by an indefinite Topic form a generic statement. Features associated with definiteness include the following: [+def] for definite NPs, [-def] for indefinite NPs, [0def] for naked nouns, [-class] for proper nouns, [+class] for common nouns, [+pro] for pronouns or pronominalized NPs, $[ \pm$ ana] for short/long anaphors, [+deic] for deictic NPs.

Here below we include the parse of a text made up of 12 sentences which has been previously parsed by the LFG parser: the discourse parser computes the Expected Topic of Discourse as well as the Main Topic simply working on the information matrix associated to each referential expression(see Grosz, 1978,1981; Webber, 1981,1983,1988). An Expected Topic becomes a Main Topic if it is reinforced by the use of a coreferring pronoun in the following sentence. A shift in main topic is structurally determined in sentence 6, or by the explicit use of a proper noun in a subsequent text: the use of a property-denoting common noun does not trigger a new Expected Topic. The algorithm is a simple finite state machine with four states: change, shift, continue, retaining. It receives as input the list of all referential expressions and then selects 
according to scoring criteria the candidates for discourse functions: potential topics may be used for lack of better candidates. Features are discussed at length in Bianchi, Delmonte(1989a,1989b): \pm MU stays for morphologically unexpressed, following Bresnan(1982).

\section{SENTENCE N. : 1}

gli,piaceva,parlare,del,suocero,come,di, una,facile,occasione, mancata, che, chiunque, altro,avrebbe,sfruttato, ma,che,lui,avveduti,preferiva,lasciare,perdere/To-him (he) liked to talk of the (about his) father-in-law as of (as if he were) an easy chance missed which anybody else would have exploited but which, him-nom (he), Avveduti, preferred to let go.

ref_express15,pred(avveduti,[+ref,-pro,-ana],3,mas,sing,human,experiencer,obj2)/20

ref_express16,pred(suocero,[+ref,-pro,-ana, +def],3,mas,sing,human,theme,obl)/43

EXPECTED TOPIC : ref_express15,pred(avveduti,[+ref,-pro,-ana],3,mas,sing,human,experiencer, obj2) POTENTIAL TOPICS : ref_express16,pred(suocero,[+ref,-pro,-ana, +def],3,mas,sing,human, theme, obl) state $(1$, change)

\section{SENTENCE N. : 2}

solo,verso,il,1950,si,rassegno_,ad,accettare,un,posto,nella,organizzazione,della,fiera,di,verona/Only towards 1950 he ended up by accepting a position in the organization(committee) of the Verona Fair. ref_express26,pred(pro,[+ref,+pro,-ana, +MU],3,sing,human,agent,subj)/10 ref_express29,pred(organizzazione,[+ref,-pro,-ana,+def],3,fem,sing,institution,locative, adjnct) /60 ref_express30,pred(fiera,[+ref,-pro,-ana, +def],3,fem,sing,event,theme,adjnct)/63 ref_express23,pred(1950,[+ref,-pro,-ana, +def],3,mas,sing,time,locative,adjnct)/1050 ref_express31,pred(verona,[+ref,-pro,-ana],3,fem,sing,place,locative,adjnct)/1080 ref_express26: avveduti

MAIIN TOPIC : ref_express,pred(avveduti,3,mas,sing,human)

POTENTIAL TOPICS : ref_express29,pred(organizzazione,[+ref,-pro,-ana,+def],3,fem,sing institution, locative, adjnct)

ref_express30,pred(fiera,[+ref,-pro,-ana, +def],3,fem,sing,event,theme,adjnct)

ref_express23,pred(1950,[+ref,-pro,-ana, +def],3,mas,sing,time,locative,adjnct)

ref_express31,pred(verona,[+ref,-pro,-ana],3,fem,sing,place,locative,adjnct)

state $(2$, continue $)$

\section{SENTENCE N. : 3}

lo,nominarono,delegato,cioe_,una,specie,di,funzionario,viaggiante,con,incarichi,diplomatici,di,tenere, i,rapp orti, con, le,delegazioni,commerciali,',',curare,i,produttori,stranieri, ',',le,grandi,ditte, ',',la, stampa/He was appointed delegate, i.e. a kind of travelling officer with diplomatic charges, to have relations with commercial committees, take care of foreign producers, the big corporations, the press.

ref_express23,pred(pro,[+ref,+pro,-ana, +MU],3,plur,human,agent,subj)/20

ref_express24,pred(lo,[+ref,+pro,+ana,-MU],3,mas,sing,human,patient,obj)/24

ref_express26,pred(specie,[+ref,-pro,-ana, -def],3,fem,sing,human,theme,adjnct)/33

ref_express242,pred(produttore,[+ref,-pro,-ana,+def],3,mas,plur,human/institution,theme, obj)/85

ref_express243,pred(ditta,[+ref,-pro,-ana, +def],3,fem,plur,institution,theme,obj)/85

ref_express244,pred(stampa,[+ref,-pro,-ana, +def],3,fem,sing,institution,theme,obj)/85

ref_express235,pred(delegazione,[+ref,-pro,-ana, +def],3,fem,plur,human/institution,theme, adjnct)/103

ref_express24:avveduti

EXPECTED TOPIC : ref_express23,pred(pro,[+ref,+pro,-ana, +MU],3,plur,human,agent,subj)

MAIN TOPIC : ref_express,pred(avveduti,3, mas,sing,human)

POTENTIAL TOPICS : ref_express26,pred(specie,[+ref,-pro,-ana, -def],3,fem,sing,human,theme, adjnct)

ref_express242,pred(produttore,[+ref,-pro,-ana, +def],3,mas,plur,human/institution,theme,obj)

ref_express243,pred(ditta,[+ref,-pro,-ana,+def],3,fem,plur,institution,theme,obj)

ref_express244,pred(stampa,[+ref,-pro,-ana,+def],3,fem,sing,institution,theme,obj)

ref_express235,pred(delegazione,[+ref,-pro,-ana,+def],3,fem,plur,human/institution,theme,adjnct) 
state(3,retaining)

\section{SENTENCE N. : 4}

un,compito,che,corrispondeva,bene, alla,sua,vocazione, e, nel, quale, avveduti,sapeva, giostrare, con, notevole, a gilita_/a task which fitted well with his bent/inclination, and in which Avveduti could joust with remarkable agility(Avveduti felt entirely at his ease).

ref_express15,pred(avveduti,+ref,-pro,-ana,3,mas,sing,human,agent,subj)/20

ref_express9,pred(suo,[+ref,+pro,-ana, -MU]3,sing,possessor,subj)/31

ref_express9:avveduti

MAIIN TOPIC : ref_express,pred(avveduti,3,mas,sing,human)

POTENTIAL TOPICS : ref_express9,pred(avveduti,[+ref,-pro,-ana],3,mas,sing,human,possessor, subj) state $(4$, continue $)$

\section{SENTENCE N. : 5}

quando,il,suocero,mori_egli,non,perse,il,posto/when his brother-in-law died, he did not lose his position ref_express15,pred(suocero,[+ref,-pro,-ana,+def],3,mas,sing,human,agent,subj)/20

ref_express17,pred(egli,[+ref,+pro,-ana, -MU],3, mas,sing,human,agent,subj)/20

ref_express17:avveduti

MĀIN TOPIC : ref_express,pred(avveduti,3,mas,sing,human)

POTENTIAL TOPICS : ref_express 15,pred(suocero,[+ref,-pro,-ana,+def],3,mas,sing,human,agent, subj) state $(5$, continue $)$

\section{SENTENCE N. : 6}

a,verona,il,collegio,di,alberti,lo, aveva,ereditato,trabucchi,e,col,collegio, aveva,ereditato,la,presidenza,della, fiera/nn Verona, Alberti's college Trabucchi had inherited it(had been inherited by Trabucchi), and with the college, (he) had inherited the chair of the Fair.

ref_express29,pred(trabucchi, [+ref,-pro,-ana],3,mas,sing,human,agent,fuoco)/10

ref_express27,pred(collegio,[+ref,-pro,-ana,+def],3,mas,sing,institution,theme,topic)/23

ref_express33,pred(presidenza,[+ref,-pro,-ana,+def],3,fem,sing,human/institution,theme, obj)/25

ref_express28,pred(alberti,[+ref,-pro,-ana],3,mas,sing,human,possessor,subj)/31

ref_express34,pred(fiera,[+ref,-pro,-ana,+def],3,fem,sing,event,theme,adjnct)/53

ref_express26,pred(verona,[+ref,-pro,-ana],3,fem,sing,place,locative,adjnct)/1060

MAIN TOPIC : ref_express,pred(trabucchi,3,mas,sing,human)

SECONDARY TOPIC : ref_express,pred(avveduti,3,mas,sing,human)

POTENTIAL TOPICS : ref_express27,pred(collegio,[+ref,-pro,-ana,+def],3,mas,sing,institution, theme, topic)

ref_express33,presidenza,[+ref,-pro,-ana,+def],3,fem,sing,human/istitution,theme,obj)

ref_express28,alberti,[+ref,-pro,-ana],3,mas,sing,human,possessor,subj)

ref_express34,fiera,[+ref,-pro,-ana,+def],3,fem,sing,event,theme,adjnct)

ref_express26,verona,[+ref,-pro,-ana],3,fem,sing,place,locative,adjnct)

state(6,shifting)

\section{SENTENCE N. : 7}

trabucchi,continuo_,a,valersi,della,collaborazione,di,avveduti/Trabucchi continued availing himself of Avveduti's collaboration.

ref_express3,pred(trabucchi,[+ref,-pro,-ana],3,mas,sing,human,agent,subj)/10

ref_express5,pred(avveduti,[+ref,-pro,-ana],3,mas,sing,human,agent,subj)/30

MAIN TOPIC : ref_express,pred(trabucchi,3,mas,sing,human)

SECONDARY TOPIC : ref_express,pred(avveduti,3,mas,sing,human)

POTENTIAL TOPICS : ref_express5,pred(avveduti,[+ref,-pro,-ana],3,mas,sing,human,agent,subj)

state $(7$, continue) 


\section{SENTENCE N. : 8}

lo,ufficiale,del,novara_cavalleria,gli,era,simpatico/the Novara Cavalry officer was nice to him (he liked the Novara-Cavalry officer)

ref_express117,pred(gli,[+ref,+pro,+ana, -MU],3,mas,sing,human,experiencer,obj2)/10

ref_express116,pred(novara_cavalleria,[+ref,-pro,ana,+def],3,mas,sing,institution,theme, adjnct)/43

ref_express117:trabucchi

MAIN TOPIC : ref_express,pred(,trabucchi,3,mas,sing,human)

POTENTIAL TOPICS : ref_express,pred(avveduti,3,mas,sing,human)

ref_express116,pred(novara_cavalleria,[+ref,-pro,-ana,+def],3,mas,sing,institution,theme, adjnct)

state $(8$, continue $)$

\section{SENTENCE N. : 9}

la,sua,distinzione, lo,impressionava/his distinction impressed him ref_express19,pred(lo,[+ref,+pro,+ana,-MU],3,mas,sing,human,experiencer,obj)/13

ref_express 18,pred(suo,[+ref,+pro,-ana, -MU],sing,possessor,subj)/21

ref_express18:avveduti

ref_express19:trabucchi

MÄIN TOPIC : ref_express,pred(trabucchi,3,mas,sing,human)

POTENTIAL TOPICS : ref_express 18,pred(avveduti,[+ref,+pro,-ana],3,mas,sing,human,possessor, subj) state $(9$,continue $)$

\section{SENTENCE N. : 10}

lo,confermo_,nello,incarico,alla,fiera/he confirmed him in the position at the Fair ref_express8,pred(pro,[+ref,+pro,-ana,+MU],3,sing,human,agent,subj)/10

ref_express9,pred(lo,[+ref,+pro,+ana,-MU],3,mas,sing,human,patient,obj)/14

ref_express 11,pred(fiera,[+ref,-pro,-ana, +def],3,fem,sing,event,locative,adjnct)/50

ref_express9:avveduti

ref_express8:trabucchi

MAIN TOPIC : ref_express,pred(trabucchi,3,mas,sing,human)

POTENTIAL TOPICS : ref_express9,pred(avveduti,[+ref,+pro,-ana], 3, mas, sing, human, patient, obj) ref_express11,pred(fiera,[+ref,-pro,-ana,+def],3,fem,sing,event,locative,adjnct)

state(10,continue)

\section{SENTENCE N. : 11}

avveduti,funzionava,benissimo,come,segretario,particolare/Avveduti worked very well as special secretary ref_express11,pred(avveduti,[+ref,-pro,-ana],3,mas,sing,human,agent,subj)/10

EXPECTED TOPIC : ref_express,pred(avveduti,3,mas,sing,human)

SECONDARY TOPIC : ref_express,pred(trabucchi,3,mas,sing,human)

state(11,change)

\section{SENTENCE N. : 12}

sapeva,mobilitare,prefetti,e,questori/he knew (how) to mobilize prefects and questors ref_express1,pred(pro,[+ref,+pro,-ana,-MU],3,sing,human,agent,subj)/10 ref_express38,pred(prefetto,[+ref,-pro,-ana, \pm def],3,mas,plur,human,theme,obj)/25 ref_express40,pred(questore,[+ref,-pro,-ana, \pm def],3,mas,plur,human,theme,obj)/25

ref_express1:avveduti

MAIIN TOPIC : ref_express,pred(avveduti,3,mas,sing,human)

SECONDARY TOPIC : ref_express,pred(trabucchi,3, mas,sing,human)

POTENTIAL TOPICS : ref_express38,pred(prefetto,[+ref,-pro,-ana, \pm def], 3, mas, plur, human, theme, obj) ref_express40,pred(questore,[+ref,-pro,-ana, \pm def],3,mas,plur,human,theme,obj)

state(12,continue) 


\section{Bibliography}

ALLEN J.F.(1983a), Argot: a System Overview, in N.Cercone(ed),Computational Linguistics, Vol.5, Pergamon Press, 97-109.

ALLEN J.F.(1983b), Maintaining Knowledge about Temporal Intervals, Communications of the ACM, 26,11, 832-843.

ALLEN J.F.(1984), Towards a General Theory of Action and Time, Artificial Intelligence 23, 123-154.

BIANCHI D., DELMONTE R.(1989a), Binding Pronominals with an LFG Parser, MS, University of Venice.

BIANCHI D., DELMONTE R.(1989b), From Grammatical Representation to Discourse Structure, MS, University of Venice.

BRESNAN, J. (ed)(1982), The Mental Representation of Grammatical Relations, MIT Press, Cambridge(Mass.)

BRESNAN J., KANERVA (1989), Locative Inversion in Chichewa: A Case Study of Factorization in Grammar, Linguistic Inquiry 20, 1, 1-50.

CHOMSKY N.(1977), Essays on Form and Interpretation, American Elsevier, New York.

DELMONTE R.(1985), Parsing Difficulties \& Phonological Processing in Italian, Proceedings of the 2nd

Conference of the European Chapter of ACL, Geneva, 136-145.

DELMONTE R.(1987a), Grammatica e Ambiguità in Italiano, Annali di Ca' Foscari, XXVI, 1-2, 257-333.

DELMONTE R.(1987b), The Realization of Semantic Focus and Language Modeling, in Proceedings of the

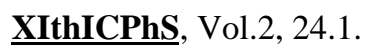

DELMONTE R.(1987c), Il principio del sottoinsieme e l'acquisizione del linguaggio, in P.Cordin(ed),Ipotesi e

Applicazioni di Teoria Linguistica, - dal XIII Incontro di Grammatica Generativa, Trento, 47-64.

DELMONTE R.(1988a), Focus and the Semantic Component, in Rivista di Grammatica Generativa, 81121.

DELMONTE R. (1988b), Appunti per un corso di Grammatica Lessico-Funzionale, Annali di Ca'Foscari, XXVII, 1-2, 51-110

DELMONTE R.(1989), From Subcategorization Frames to Thematic Roles, in R.Delmonte(ed.),Lessico, Stutture e Interpretazione, - Studi Linguistici Applicati I, Unipress, Padova, Chapt.IV,167-212.

DELMONTE R., R.DOLCI(1989b), Parsing Italian with a Context-Free Recognizer, Annali di Ca' Foscari XXVIII, 1-2,123-161.

ENÇ MÜ RVET(1986), Towards a referential analysis of temporal expressions, Linguistic and Philosophy 9 FURLAN M.(1988), Analisi delle Relazioni Aspetto-Temporale nella Frase, in R.Delmonte(ed.),Lessico, Stutture e Interpretazione, - Studi Linguistici Applicati I, Unipress, Padova, Chapt.III, 131-166.

GROSZ B.(1978), Focus Spaces: A Representation of the Focus of Attention of a Dialog, in D.Walker(ed), op.cit., $269-284$.

GROSZ B.(1981), Focusing and Description in Natural Language Dialogues, in A.Joshi, B.Webber, and I.Sag(eds), Elements of Discourse Understanding, CUP, 84-105.

HAï K I.(1984), Indirect Binding, Linguistic Inquiry 15,2,185-224.

HALVORSEN P.K.(1983), Semantics for Lexical Functional Grammar,Linguistic Inquiry 4,567-616.

HIGGINBOTHAM J.(1980), Pronouns and Bound Variables, Linguistic Inquiry 11, 679-708.

HIGGINBOTHAM J.(1983), Logical Form, Binding, and Nominals, Linguistic Inquiry 14, 3, 395-420.

HIGGINBOTHAM J.(1985), On Semantics, Linguistic Inquiry 16, 4, 547-593.

HINRICHS E.(1988), Tense, Quantifiers, and Contexts, in Computational Linguistics - Special Issue on Tense and Aspect - 14, 2, 3-14.

HOBBS J.R., SHIEBER S.M.(1987), An Algorithm for Generating Quantifier Scoping, Computational Linguistics, 13, 1-2, (January-June), 47-63.

HORNSTEIN N.(1989), As Times Goes By: Tense and Universal , ms, University of Brandeis.

IOUP G.(1975), Some Universals for Quantifier Scope,in J.P.Kimball(ed),Syntax and Semantics 4., Academic Press, New York, 37-58.

JACKENDOFF R.(1976), Towards an Explanatory Semantic Representation, Linguistic Inquiry 7, 89-150

JACKENDOFF R. (1985) Believing and Intending: Two Sides of the Same Coin, Linguistic Inquiry 16, 445460 . 
MAY R.(1985), Logical Form, The MIT Press, Cambridge(Mass.).

MAY R.(1989), Interpreting Logical Form, Linguistics and Philosophy 12, 4, 387-436.

ter MEULEN A.(1983), The representation of time in natural language, in A. ter Meulen(ed), $\underline{\text { Studies in }}$ Modeltheoretic Semantics, Foris, Dordrecht, 177-192.

ROBERTS I.(1986), The Representation of Implicit and Dethematized Subjects, Foris, Dordrecht.

SANSON A.(1990), Die Derivation der linguistichen Inferenzen im Satz durch die Eigenschaften der Prä dikatArgument Strukturen, Unpublished Doctoral Dissertation. University of Venice.

SIDNER C.(1983), Focusing in the comprehension of definite anaphora, in M.Brady, R.Berwick(1983)(eds.), Computational Models of Discourse, MIT Press, Cambridge(Mass.), 267-330.

WEBBER B.(1981), Discourse model synthesis: preliminaries to reference in A.Joshi, B.Webber, and I.Sag(eds), Elements of Discourse Understanding, CUP,

WEBBER B.(1983), So what can we talk about now?, in Brady M. and Berwick R.(eds), Computational Models of Discourse, The MIT Press, 331-372.

WEBBER B.(1988), Tense as Discourse Anaphor, in Computational Linguistics - Special Issue on Tense and Aspect - 14, 2, 61-73.

WILLIAMS E.(1986), A Reassignment of the Functions of LF, Linguistic Inquiry 17, 2, 265-299.

ZAENEN A.(1983), On Syntactic Binding, Linguistic Inquiry 14, 3, 469-504. 